\title{
CALCULATION OF THE HORIZONTAL TWO-DIMENSIONAL UNSTEADY FLOWS BY THE METHOD OF CHARACTERISTICS
}

\author{
Tran Gia Lich, Nguyen Minh Son and Le viet Cuong \\ Institute of Mathematics, Hanoi, Vietnam
}

\begin{abstract}
This paper will be concerned with the characteristic form of the twodimensional Saint-Venant equation system, the supplementary equations at the boundaries, the methods of characteristics for solving the equation system and some numerical experiments.
\end{abstract}

\section{Two-dimensional Saint-Venant equation system}

The Saint-Venant equation system describing horizontal two-dimensional unsteady flow without turbulent diffusion components can be written in the following form (see $[1,2])$ :

$$
\begin{aligned}
& \frac{\partial u}{\partial t}+u \frac{\partial u}{\partial x}+v \frac{\partial u}{\partial y}+g \frac{\partial H}{\partial x}=\phi_{1} \\
& \frac{\partial v}{\partial t}+u \frac{\partial v}{\partial x}+v \frac{\partial v}{\partial y}+g \frac{\partial H}{\partial y}=\phi_{2} \\
& \frac{\partial H}{\partial t}+\frac{\partial H u}{\partial x}+\frac{\partial H v}{\partial y}=0 .
\end{aligned}
$$

where:

$$
\begin{aligned}
& \phi_{1}={ }^{\natural} v-g \frac{\sqrt{u^{2}+v^{2}}}{C_{s}^{2} H} u+\frac{\tau_{x}}{\rho H}+g \frac{\partial h}{\partial x}, \\
& \phi_{2}=-\ell u-g \frac{\sqrt{u^{2}+v^{2}}}{C_{s}^{2} H} v+\frac{\tau_{y}}{\rho H}+g \frac{\partial h}{\partial y}, \\
& H=h+\zeta,
\end{aligned}
$$

$x, y$ are coordinate axes,

$t$ - time, $H$ - water depth,

$u, v$ - velocity components,

$\rho$ - water density, $g$ - gravity acceleration,

$\tau_{x}, \tau_{y}$ - components of wind stress on water surface,

$h$ - bottom depth, 
$\zeta$ - elevation of the free surface,

$C_{s}$ - Chezy's coefficient, $\ell$ - Coriolis parameter.

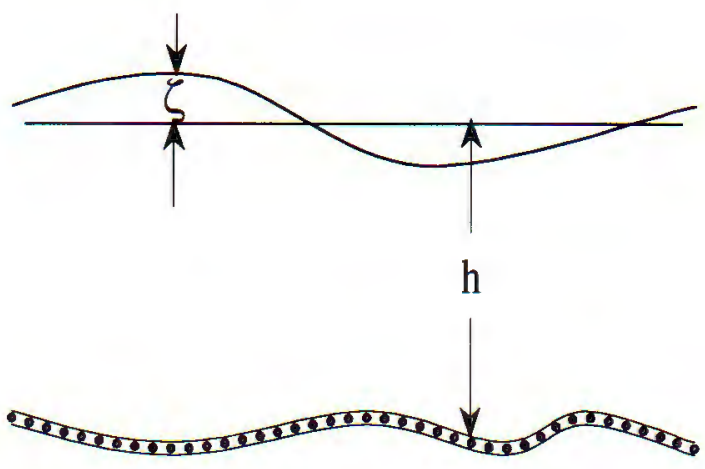

Fig. 1

The vector form of equation system (1.1) is

$$
\frac{\partial \mathbf{U}}{\partial t}+\mathbf{A} \frac{\partial \mathbf{U}}{\partial x}+\mathbf{B} \frac{\partial \mathbf{U}}{\partial y}=\phi,
$$

where

$$
\mathbf{U}=\left(\begin{array}{c}
u \\
v \\
H
\end{array}\right), \quad \boldsymbol{\phi}=\left(\begin{array}{c}
\phi_{1} \\
\phi_{2} \\
0
\end{array}\right), \quad \mathbf{A}=\left(\begin{array}{ccc}
u & 0 & g \\
o & u & 0 \\
H & 0 & u
\end{array}\right), \quad \mathbf{B}=\left(\begin{array}{ccc}
v & 0 & 0 \\
0 & v & g \\
0 & H & v
\end{array}\right)
$$

By some transformation of the unknown vector $\mathrm{U}$, the equation (1.2) can be written in the symmetric form:

$$
\frac{\partial \mathbf{V}}{\partial t}+\tilde{\mathbf{A}} \frac{\partial \mathbf{V}}{\partial x}+\widetilde{\mathbf{B}} \frac{\partial \mathbf{V}}{\partial y}=\tilde{\boldsymbol{\phi}},
$$

where:

$$
\mathbf{V}=\left(\begin{array}{c}
u \\
v \\
2 c
\end{array}\right), \quad \widetilde{\mathbf{A}}=\left(\begin{array}{ccc}
u & 0 & c \\
0 & u & 0 \\
c & 0 & u
\end{array}\right), \quad \widetilde{\mathbf{B}}=\left(\begin{array}{ccc}
v & 0 & 0 \\
0 & v & c \\
0 & c & v
\end{array}\right), \quad c=\sqrt{g H} .
$$

The equation systems (1.1), (1.2), (1.3) are quasi-linear hyperbolic. In fact, for any real values $s_{1}, s_{2}$ satisfying $s_{1}^{2}+s_{2}^{2} \neq 0$, the matrix $\mathbf{A}_{*}=s_{1} \mathbf{A}+s_{2} \mathbf{B}$ has three different real eigen values $\lambda_{j}$ as follows:

$$
\begin{aligned}
& \lambda_{1}=w=s_{1} u+s_{2} v, \\
& \lambda_{2}=w+\sqrt{\left(s_{1}^{2}+s_{2}^{2}\right) g H}, \\
& \lambda_{3}=w-\sqrt{\left(s_{1}^{2}+s_{2}^{2}\right) g H} .
\end{aligned}
$$


It is well known (see $[1,2,5,6]$ ) that the boundary problem of linear symmetric hyperbolic equation system:

$$
\mathbf{D} \frac{\partial \mathbf{V}}{\partial t}+\overline{\mathbf{A}} \frac{\partial \mathbf{V}}{\partial x}+\overline{\mathbf{B}} \frac{\partial \mathbf{V}}{\partial y}=\bar{\phi},
$$

where $\mathbf{D}, \overline{\mathbf{A}}, \overline{\mathbf{B}}$ are symmetric matrixs and $D>0$, has a solution if beside of the initial conditions, the number of given boundary conditions is equal to the one of negative eigen values $\lambda_{j}$ of the matrix

$$
\mathbf{A}_{n}=n_{x} \overline{\mathbf{A}}+n_{y} \overline{\mathbf{B}}
$$

In addition, the equation system (1.4) has an unique solution, continuously depending on the initial conditions and its right hand side, if the boundary conditions are dissipative.

The boundary condition is said to be dissipative if any vector $\mathbf{V}$ satisfying this condition also satisfies the following inequality:

$$
\iint_{S}\left(\left[n_{t} \mathbf{D}+m_{x} \overline{\mathbf{A}}+m_{y} \overline{\mathbf{B}}\right] \mathbf{V}, \mathbf{V}\right) d s \geq 0,
$$

where $S=\partial G \times(0, T)$

$\partial G$ is the boundary of the region of consideration $G$

$\left(n_{t}, n_{x}, n_{y}\right)$ is the external normal vector of the surface $S$.

Let the boundary $\partial G$ be fixed, then $n_{t}=0$ and the inequality (1.5) may be replaced by the following inequality

$$
\left(\mathbf{A}_{n} \mathbf{V}, \mathbf{V}\right)=\left(\left[n_{x} \overline{\mathbf{A}}+n_{y} \overline{\mathbf{B}}\right] \mathbf{V}, \mathbf{V}\right) \geq 0 .
$$

Linearising the Saint-Venant equation system (1.3) we can get the linear equation system (1.4), where:

$$
\mathbf{D}=\left(\begin{array}{lll}
1 & 0 & 0 \\
0 & 1 & 0 \\
0 & 0 & 1
\end{array}\right), \quad \overline{\mathbf{A}}=\left(\begin{array}{ccc}
\bar{u} & 0 & \bar{c} \\
0 & \bar{u} & 0 \\
\bar{c} & 0 & \bar{u}
\end{array}\right), \quad \overline{\mathbf{B}}=\left(\begin{array}{ccc}
\bar{v} & 0 & 0 \\
0 & \bar{v} & \bar{c} \\
0 & \bar{c} & \bar{v}
\end{array}\right)
$$

and $\bar{u}, \bar{v}, \bar{c}, \bar{\phi}$ are known values, respectively equal to the $u, v, c, \phi$ at the previous time step $t_{k}$.

The eigen values $\lambda_{j}$ of the matrix $\mathbf{A}_{n}=n_{x} \overline{\mathbf{A}}+n_{y} \overline{\mathbf{B}}$ are

$$
\begin{aligned}
& \bar{\lambda}_{1}=\bar{w}_{n}=n_{x} \bar{u}+n_{y} \bar{v}, \\
& \bar{\lambda}_{2}=\bar{w}_{n}+\bar{c}, \\
& \bar{\lambda}_{3}=\bar{w}_{n}-\bar{c} .
\end{aligned}
$$


From this, the dissipative boundary conditions which are necessary to be given on the boundaries could be deduced as follows (see [8])

- If $-\bar{c} \leq \bar{w}_{n}<0$ : It is necessary to give two boundary conditions, which may be

$$
\begin{aligned}
& w_{s}=\psi(x, y, t), \\
& w_{n}-2 c=\xi(x, y, t),
\end{aligned}
$$

where $w_{n}$ and $w_{s}$ are the projections of the velocity vector on the external normal and tangent vectors.

- If $\bar{w}_{n}<-\bar{c}$ : The three boundary conditions are needed:

$$
w_{n}=\varphi(x, y, t), \quad w_{s}=\psi(x, y, t), \quad H=f(x, y, t) .
$$

- If $0<\bar{w}_{n}<\bar{c}$ : It is necessary to give only one boundary condition, which may be one of following

$$
\begin{aligned}
w_{n} & =\varphi(x, y, t), \\
H & =Z(x, y, t), \\
w_{n}-2 c & =\xi(x, y, t) .
\end{aligned}
$$

- If $\bar{c} \leq \bar{w}_{n}$ : No boundary condition is needed.

- At the solide boundary $\left(\bar{w}_{n}=0\right)$, it is necessary to give only one boundary condition. It may be one of the following

$$
\begin{aligned}
w_{n} & =0, \\
H & =Z(x, y, t), \\
w_{n}-2 c & =\xi(x, y, t) .
\end{aligned}
$$

In the simple case, when the flow is very slow and the convective components $\left(u \frac{\partial u}{\partial x}+v \frac{\partial u}{\partial y}\right),\left(u \frac{\partial v}{\partial x}+v \frac{\partial v}{\partial y}\right)$ are very small in comparison with the other ones, we have the modified Saint-Venant equation system:

$$
\frac{\partial \mathbf{V}}{\partial t}+\mathbf{A}_{M} \frac{\partial \mathbf{V}}{\partial x}+\mathbf{B}_{M} \frac{\partial \mathbf{V}}{\partial y}=\boldsymbol{\phi}
$$

where

$$
\mathbf{A}_{M}=\left(\begin{array}{ccc}
0 & 0 & c \\
0 & 0 & 0 \\
c & 0 & u
\end{array}\right), \quad \mathbf{B}_{M}=\left(\begin{array}{ccc}
0 & 0 & 0 \\
0 & 0 & c \\
0 & c & v
\end{array}\right)
$$

Equation system (1.6) is also quasi-linear hyperbolic. 
The matrix $A_{M}=n_{x} \mathbf{A}_{M}+n_{y} \mathbf{B}_{M}$ has three different real eigen values

$$
\begin{aligned}
& \lambda_{M 1}=0, \\
& \lambda_{M 2}=\frac{w_{n}+\sqrt{w_{n}^{2}+4 g H}}{2}>0, \\
& \lambda_{M 3}=\frac{w_{n}-\sqrt{w_{n}^{2}+4 g H}}{2}<0 .
\end{aligned}
$$

Therefore, for the linearized boundary problem (1.6) at boundary of any kinds (liquid, solid, inflow, outflow) only one dissipative boundary condition is needed.

- If $\bar{w}_{n}<0$ it may be

$$
H=Z(x, y, t) .
$$

- If $0<\bar{w}_{n}$ it may be one of the following two conditions

$$
\begin{aligned}
H & =Z(x, y, t), \\
w_{n} & =\varphi(x, y, t) .
\end{aligned}
$$

- At the solid boundary $\left(\bar{w}_{n}=0\right)$ it may be one of the following two conditions:

$$
\begin{aligned}
w_{n} & =0, \\
H & =Z(x, y, t) .
\end{aligned}
$$

\section{Supplementary equations on the boundary}

In order to determine three unknown functions $u, v$ and $H$ at the boundaries, where only one or two boundary conditions are given, it is necessary to establish some supplementary equations, which combining with the given boundary condition give a closed system for determining the functions $u, v, H$ at the boundaries.

Let $\boldsymbol{\tau}_{i}=\left(\tau_{i 1}, \tau_{i 2}, \tau_{i 3}\right),(i=1,2,3)$ be left eigen vectors of the matrix $\mathbf{A}$ and $\sigma_{i}$ its left eigen values, then we have:

$$
\begin{aligned}
\tau_{i} \mathbf{A} & =\sigma_{i} \tau_{i}, \\
\tau_{i}\left(\frac{\partial \mathbf{U}}{\partial t}+\mathbf{A} \frac{\partial \mathbf{U}}{\partial x}\right) & =\left(\tau_{i} \frac{\partial \mathbf{U}}{\partial t}+\tau_{i} \mathbf{A} \frac{\partial \mathbf{U}}{\partial x}\right)=\left(\tau_{i} \frac{\partial \mathbf{U}}{\partial t}+\sigma_{i} \tau_{i} \frac{\partial \mathbf{U}}{\partial x}\right), \\
& =\tau_{i}\left(\frac{\partial \mathbf{U}}{\partial t}+\sigma_{i} \frac{\partial \mathbf{U}}{\partial x}\right)=\tau_{i}\left(\frac{d \mathbf{U}}{d t}\right)_{x_{i}}
\end{aligned}
$$

where:

$$
\left(\frac{d \mathbf{U}}{d t}\right)_{x_{i}}=\frac{\partial \mathbf{U}}{\partial t}+\sigma_{i} \frac{\partial \mathbf{U}}{\partial x}
$$


Denote that:

$$
\boldsymbol{\Omega}=\left(\begin{array}{l}
\tau_{1} \\
\tau_{2} \\
\tau_{3}
\end{array}\right)=\left(\begin{array}{lll}
\tau_{11} & \tau_{12} & \tau_{13} \\
\tau_{21} & \tau_{22} & \tau_{23} \\
\tau_{31} & \tau_{32} & \tau_{33}
\end{array}\right)
$$

from (1.2), we will have the following equation:

$$
\begin{aligned}
\mathbf{B} \frac{\partial \mathbf{U}}{\partial y} & =\boldsymbol{\phi}-\left(\frac{\partial \mathbf{U}}{\partial t}+\mathbf{A} \frac{\partial \mathbf{U}}{\partial x}\right)=\boldsymbol{\phi}-\boldsymbol{\Omega}^{-1} \cdot \mathbf{\Omega}\left(\frac{\partial \mathbf{U}}{\partial t}+\mathbf{A} \frac{\partial \mathbf{U}}{\partial x}\right) \\
& =\phi-\Omega^{-1}\left\|\tau_{i}\left(\frac{d \mathbf{U}}{d t}\right)_{x_{i}}\right\|
\end{aligned}
$$

where:

$$
\left\|\tau_{i}\left(\frac{d \mathrm{U}}{d t}\right)_{x_{i}}\right\|=\left(\begin{array}{c}
\tau_{1}\left(\frac{d \mathbf{U}}{d t}\right)_{x_{1}} \\
\tau_{2}\left(\frac{d \mathbf{U}}{d t}\right)_{x_{2}} \\
\tau_{3}\left(\frac{d \mathbf{U}}{d t}\right)_{x_{3}}
\end{array}\right)
$$

By an analogous argument we have:

$$
\mathbf{A} \frac{\partial \mathbf{U}}{\partial x}=\boldsymbol{\phi}-\mathbf{W}^{-1}\left\|\boldsymbol{\nu}_{j}\left(\frac{d \mathbf{U}}{d t}\right)_{y_{j}}\right\|
$$

where $\nu_{j}=\left(\nu_{j_{1}}, \nu_{j_{2}}, \nu_{j_{3}}\right),(j=1,2,3)$ are left eigen vectors and $\mu_{i}$ are left eigen values of the matrix $B$.

$$
\begin{gathered}
\left(\frac{d \mathbf{U}}{d t}\right)_{y_{j}}=\frac{\partial \mathbf{U}}{\partial t}+\mu_{j} \frac{\partial \mathbf{U}}{\partial y} \\
\mathbf{W}=\left(\begin{array}{l}
\nu_{1} \\
\nu_{2} \\
\nu_{3}
\end{array}\right)=\left(\begin{array}{lll}
\nu_{11} & \nu_{12} & \nu_{13} \\
\nu_{21} & \nu_{22} & \nu_{23} \\
\nu_{31} & \nu_{32} & \nu_{33}
\end{array}\right), \quad\left\|\nu_{j}\left(\frac{d \mathbf{U}}{d t}\right)_{y_{j}}\right\|=\left(\begin{array}{l}
\nu_{1}\left(\frac{d \mathbf{U}}{d t}\right)_{y_{1}} \\
\nu_{2}\left(\frac{d \mathbf{U}}{d t}\right)_{y_{2}} \\
\nu_{3}\left(\frac{d \mathbf{U}}{d t}\right)_{y_{3}}
\end{array}\right) .
\end{gathered}
$$

Putting (2.1) and (2.2) into (1.2), we obtain (see [3]):

$$
\frac{\partial \mathbf{U}}{\partial t}+\phi-\mathbf{\Omega}^{-1}\left\|\tau_{1}\left(\frac{d \mathbf{U}}{d t}\right)_{x_{i}}\right\|-\mathbf{W}^{-1}\left\|\boldsymbol{\nu}_{j}\left(\frac{d \mathbf{U}}{d t}\right)_{y_{j}}\right\|=0 .
$$

Equation system (2.3) may be rewritten in the explicit form: 


$$
\begin{aligned}
\frac{\partial u}{\partial t} & -\left(\frac{d u}{d t}\right)_{y_{1}}-\frac{1}{2}\left(\frac{d u}{d t}\right)_{x_{2}}-\frac{c}{2 H}\left(\frac{d H}{d t}\right)_{x_{2}}-\frac{1}{2}\left(\frac{d u}{d t}\right)_{x_{3}}+\frac{c}{2 H}\left(\frac{d H}{d t}\right)_{x_{3}}+\phi_{1}=0 \\
\frac{\partial v}{\partial t} & -\left(\frac{d v}{d t}\right)_{x_{1}}-\frac{1}{2}\left(\frac{d v}{d t}\right)_{y_{2}}-\frac{c}{2 H}\left(\frac{d H}{d t}\right)_{y_{2}}-\frac{1}{2}\left(\frac{d v}{d t}\right)_{y_{3}}+\frac{c}{2 H}\left(\frac{d H}{d t}\right)_{y_{3}}+\phi_{2}=0 \\
\frac{\partial H}{\partial t} & -\frac{H}{2 c}\left(\frac{d u}{d t}\right)_{x_{2}}-\frac{1}{2}\left(\frac{d H}{d t}\right)_{x_{2}}+\frac{H}{2 c}\left(\frac{d u}{d t}\right)_{x_{3}}-\frac{1}{2}\left(\frac{d H}{d t}\right)_{x_{3}} \\
& -\frac{H}{2 c}\left(\frac{d v}{d t}\right)_{y_{2}}-\frac{1}{2}\left(\frac{d H}{d t}\right)_{y_{2}}+\frac{H}{2 c}\left(\frac{d v}{d t}\right)_{y_{3}}-\frac{1}{2}\left(\frac{d H}{d t}\right)_{y_{3}}=0
\end{aligned}
$$

where:

$$
\begin{array}{lll}
\sigma_{1}=u, & \sigma_{2}=u+c, & \sigma_{3}=u-c, \\
\mu_{1}=v, & \mu_{2}=v+c, & \mu_{3}=v-c .
\end{array}
$$

Let the boundary of the region of consideration $G$ be the line segments parallel to the coordinate axes $O x$ and $O y$ (see Fig. 2). Then from (2.4), it is easy to deduce the supplementary equation at different boundaries. They are differential equations containing only the derivatives $\left(\frac{d}{d t}\right)_{x_{i}}$ and $\left(\frac{d}{d t}\right)_{y_{j}}$ with respect to the directions $x_{i}$ and $y_{j}$, which meet the hyperplane $t_{k}$ at the points in the region $G$ :

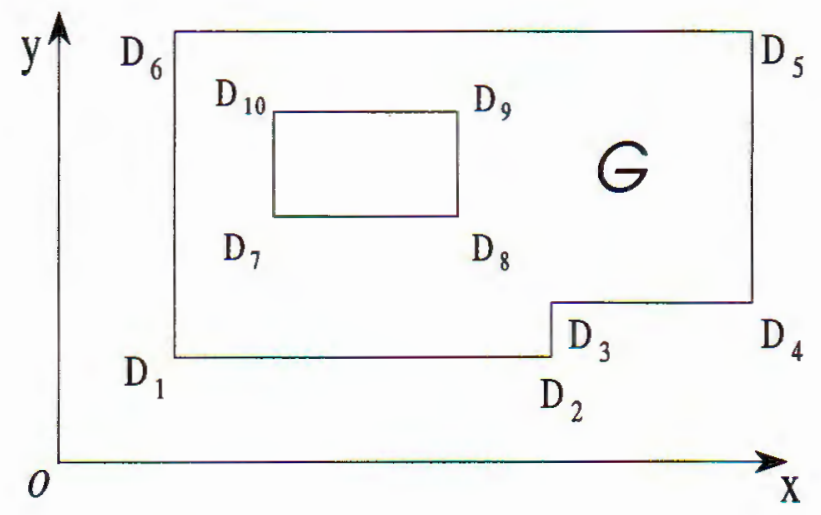

Fig. 2

A) For the inflow boundary $\left(w_{n}<0\right)$

1) If: $c<\left|w_{n}\right|$, no supplementary equation is needed.

2) If $\left|w_{n}\right| \leq c$, it is necessary to give only one supplementary equation, which is:

a) At the left boundaries $D_{1} D_{6}, D_{8} D_{9}$

$$
\begin{aligned}
\frac{\partial H}{\partial t} & +\frac{H}{c}\left(\frac{d u}{d t}\right)_{x_{3}}-\left(\frac{d H}{d t}\right)_{x_{3}}-\frac{H}{2 c}\left(\frac{d v}{d t}\right)_{y_{2}}-\frac{1}{2}\left(\frac{d H}{d t}\right)_{y_{2}}+\frac{H}{2 c}\left(\frac{d v}{d t}\right)_{y_{3}} \\
& -\frac{1}{2}\left(\frac{d H}{d t}\right)_{y_{3}}-\frac{H}{c} \phi_{1}-\frac{H}{c} \frac{\partial u}{\partial t}+\frac{H}{c}\left(\frac{d u}{d t}\right)_{y_{1}}=0 .
\end{aligned}
$$


b) At the right boundaries $D_{2} D_{3}, D_{4} D_{5}, D_{7} D_{10}$

$$
\begin{aligned}
\frac{\partial H}{\partial t} & -\frac{H}{c}\left(\frac{d u}{d t}\right)_{x_{2}}-\left(\frac{d H}{d t}\right)_{x_{2}}-\frac{H}{2 c}\left(\frac{d v}{d t}\right)_{y_{2}}-\frac{1}{2}\left(\frac{d H}{d t}\right)_{y_{2}}+\frac{H}{2 c}\left(\frac{d v}{d t}\right)_{y_{3}} \\
& -\frac{1}{2}\left(\frac{d H}{d t}\right)_{y_{3}}+\frac{H}{c} \phi_{1}+\frac{H}{c} \frac{\partial u}{\partial t}-\frac{H}{c}\left(\frac{d u}{d t}\right)_{y_{1}}=0 .
\end{aligned}
$$

c) At the lower boundaries $D_{1} D_{2}, D_{3} D_{4}, D_{9} D_{10}$

$$
\begin{aligned}
\frac{\partial H}{\partial t} & -\frac{H}{2 c}\left(\frac{d u}{d t}\right)_{x_{2}}-\frac{1}{2}\left(\frac{d H}{d t}\right)_{x_{2}}+\frac{H}{2 c}\left(\frac{d u}{d t}\right)_{x_{3}}-\frac{1}{2}\left(\frac{d H}{d t}\right)_{x_{3}}+\frac{H}{c}\left(\frac{d v}{d t}\right)_{y_{3}} \\
& -\left(\frac{d H}{d t}\right)_{y_{3}}-\frac{H}{c} \phi_{2}-\frac{H}{c} \frac{\partial v}{\partial t}+\frac{H}{c}\left(\frac{d v}{d t}\right)_{x_{1}}=0 .
\end{aligned}
$$

d) At the upper boundaries $D_{5} D_{6}, D_{7} D_{8}$

$$
\begin{aligned}
\frac{\partial H}{\partial t} & -\frac{H}{2 c}\left(\frac{d u}{d t}\right)_{x_{2}}-\frac{1}{2}\left(\frac{d H}{d t}\right)_{x_{2}}+\frac{H}{2 c}\left(\frac{d u}{d t}\right)_{x_{3}}-\frac{1}{2}\left(\frac{d H}{d t}\right)_{x_{3}}-\frac{H}{c}\left(\frac{d v}{d t}\right)_{y_{2}} \\
& -\left(\frac{d H}{d t}\right)_{y_{2}}+\frac{H}{c} \phi_{2}+\frac{H}{c} \frac{\partial v}{\partial t}-\frac{H}{c}\left(\frac{d v}{d t}\right)_{x_{1}}=0 .
\end{aligned}
$$

B) For the outflow or solid boundaries $\left(w_{n} \geq 0\right)$

1) If $w_{n} \geq c$, all three equations of the system (2.4) are supplementary equations.

2) If $w_{n}<c$, two supplementary equations are needed. They are:

a) At the left boundaries:

i. Equation (2.5)

ii. $\frac{\partial v}{\partial t}-\left(\frac{d v}{d t}\right)_{x_{1}}-\frac{1}{2}\left(\frac{d v}{d t}\right)_{y_{2}}-\frac{c}{2 H}\left(\frac{d H}{d t}\right)_{y_{2}}-\frac{1}{2}\left(\frac{d v}{d t}\right)_{y_{3}}+\frac{c}{2 H}\left(\frac{d H}{d t}\right)_{y_{3}}+\phi_{2}=0$.

b) At the right boundaries

i. Equation (2.6)

ii. Equation (2.9)

c) At the lower boundaries

i. Equation (2.7)

ii. $\frac{\partial u}{\partial t}-\left(\frac{d u}{d t}\right)_{y_{1}}-\frac{1}{2}\left(\frac{d u}{d t}\right)_{x_{2}}-\frac{c}{2 H}\left(\frac{d H}{d t}\right)_{x_{2}}-\frac{1}{2}\left(\frac{d u}{d t}\right)_{x_{3}}+\frac{c}{2 H}\left(\frac{d H}{d t}\right)_{x_{3}}+\phi_{1}=0$

d) At the upper boundaries

i. Equation (2.8)

ii. Equation (2.10) 
C) For the equation system (1.6), at the boundaries, two supplementary equations are always needed. They are:

1) At the left boundaries

i. $\phi_{2}-\frac{\nabla-v}{2 \nabla}\left[\left(\frac{d v}{d t}\right)_{y_{2}}+\frac{v+\nabla}{2 H}\left(\frac{d H}{d t}\right)_{y_{2}}\right]-\frac{\nabla+v}{2 \nabla}\left[\left(\frac{d v}{d t}\right)_{y_{3}}+\frac{v-\nabla}{2 H}\left(\frac{d H}{d t}\right)_{y_{3}}\right]=0$

ii. $\frac{\partial H}{\partial t}+\frac{2 H}{\Delta-u}\left[\left(\frac{d u}{d t}\right)_{x_{3}}+\frac{u-\nabla}{2 H}\left(\frac{d H}{d t}\right)_{x_{3}}\right]-\frac{H}{\nabla}\left[\left(\frac{d v}{d t}\right)_{y_{2}}+\frac{v+\nabla}{2 H}\left(\frac{d H}{d t}\right)_{y_{2}}\right]$

$$
+\frac{H}{\nabla}\left[\left(\frac{d v}{d t}\right)_{y_{3}}+\frac{v-\nabla}{2 H}\left(\frac{d H}{d t}\right)_{y_{3}}\right]-\frac{2 H}{\Delta-u} \phi_{1}=0 .
$$

2) At the right boundaries

i. Equation (2.11)

ii. $\frac{\partial H}{\partial t}-\frac{2 H}{\Delta+u}\left[\left(\frac{d u}{d t}\right)_{x_{2}}+\frac{u+\nabla}{2 H}\left(\frac{d H}{d t}\right)_{x_{2}}\right]-\frac{H}{\nabla}\left[\left(\frac{d v}{d t}\right)_{y_{2}}+\frac{v+\nabla}{2 H}\left(\frac{d H}{d t}\right)_{y_{2}}\right]$

$$
+\frac{H}{\nabla}\left[\left(\frac{d v}{d t}\right)_{y_{3}}+\frac{v-\nabla}{2 H}\left(\frac{d H}{d t}\right)_{y_{3}}\right]+\frac{2 H}{\Delta+u} \phi_{1}=0 .
$$

3) At the lower boundaries

i. $\phi_{1}-\frac{\Delta-u}{2 \Delta}\left[\left(\frac{d u}{d t}\right)_{x_{2}}+\frac{u+\Delta}{2 H}\left(\frac{d H}{d t}\right)_{x_{2}}\right]-\frac{u+\Delta}{2 \Delta}\left[\left(\frac{d u}{d t}\right)_{x_{3}}+\frac{u-\Delta}{2 H}\left(\frac{d H}{d t}\right)_{x_{3}}\right]=0$

ii. $\frac{\partial H}{\partial t}-\frac{H}{\Delta}\left[\left(\frac{d u}{d t}\right)_{x_{2}}+\frac{u+\Delta}{2 H}\left(\frac{d H}{d t}\right)_{x_{2}}\right]+\frac{H}{\Delta}\left[\left(\frac{d u}{d t}\right)_{x_{3}}+\frac{u-\Delta}{2 H}\left(\frac{d H}{d t}\right)_{x_{3}}\right]$

$$
+\frac{2 H}{\nabla-v}\left[\left(\frac{d v}{d t}\right)_{y_{3}}+\frac{v-\nabla}{2 H}\left(\frac{d H}{d t}\right)_{y_{3}}\right]-\frac{2 H}{\nabla-v} \phi_{2}=0 .
$$

4) At the upper boundaries

i. Equation (2.14)

$$
\text { ii. } \begin{aligned}
\frac{\partial H}{\partial t} & -\frac{H}{\Delta}\left[\left(\frac{d u}{d t}\right)_{x_{2}}+\frac{u+\Delta}{2 H}\left(\frac{d H}{d t}\right)_{x_{2}}\right]+\frac{H}{\Delta}\left[\left(\frac{d u}{d t}\right)_{x_{3}}+\frac{u-\Delta}{2 H}\left(\frac{d H}{d t}\right)_{x_{3}}\right] \\
& -\frac{2 H}{\nabla+v}\left[\left(\frac{d v}{d t}\right)_{y_{2}}+\frac{v+\nabla}{2 H}\left(\frac{d H}{d t}\right)_{y_{2}}\right]+\frac{2 H}{\nabla+v} \phi_{2}=0
\end{aligned}
$$

where $\Delta=\sqrt{u^{2}+4 g H}, \nabla=\sqrt{v^{2}+4 g H}$

\section{Algorithm}

Assume that the region of consideration $G$ is divided into equal rectangles with the sides $\Delta x$ and $\Delta y$. 
The value $\Delta t$ is chosen so that

$$
\Delta t \leq \min \left\{\frac{\Delta x}{|u|+c}, \frac{\Delta y}{|v|+c}\right\} .
$$

Derivatives $\left(\frac{d f}{d t}\right)_{x_{i}}$ and $\left(\frac{d f}{d t}\right)_{y_{j}}$ are approximated by the following difference expressions (see Fig. 3):

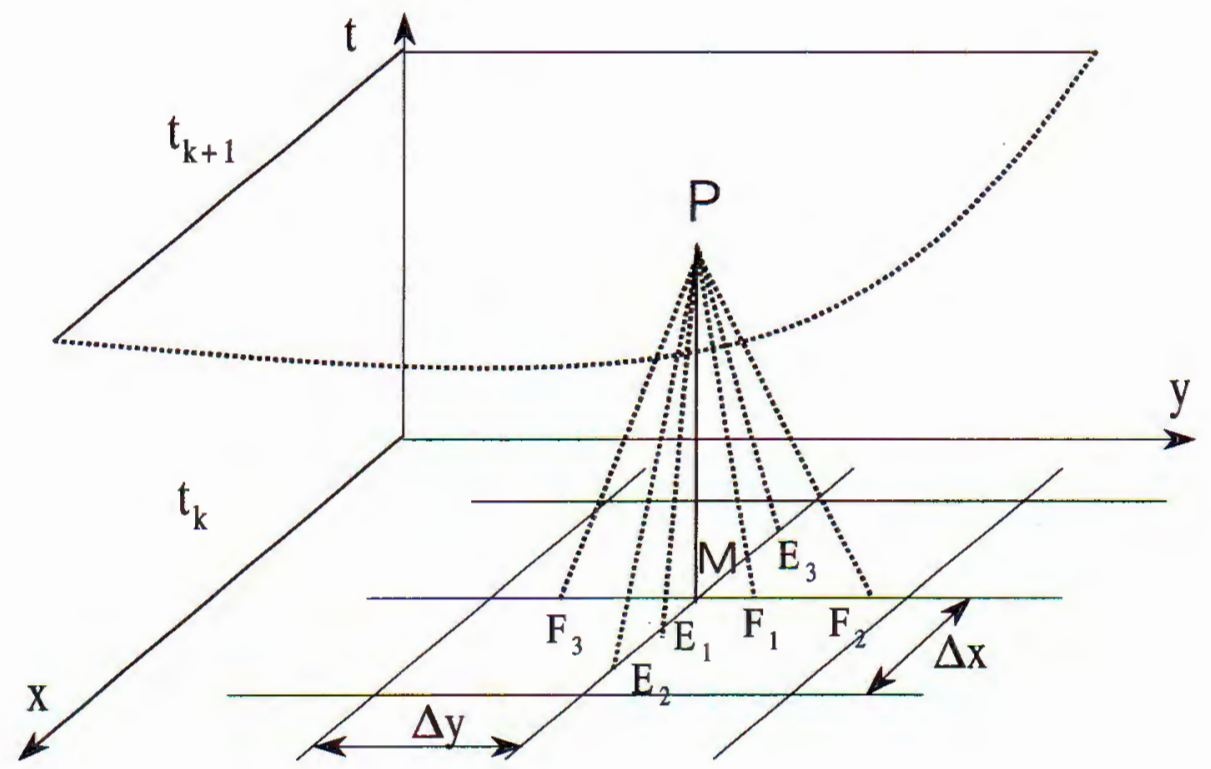

Fig. 3

$$
\begin{gathered}
\frac{\partial f}{\partial t}=\frac{f(P)-f(M)}{\Delta t} \\
\left(\frac{d f}{d t}\right)_{x_{1}}=\frac{f(P)-f\left(E_{1}\right)}{\Delta t}, \quad\left(\frac{d f}{d t}\right)_{x_{2}}=\frac{f(P)-f\left(E_{2}\right)}{\Delta t}, \quad\left(\frac{d f}{d t}\right)_{x_{3}}=\frac{f(P)-f\left(E_{3}\right)}{\Delta t}, \\
\left(\frac{d f}{d t}\right)_{y_{1}}=\frac{f(P)-f\left(F_{1}\right)}{\Delta t}, \quad\left(\frac{d f}{d t}\right)_{y_{2}}=\frac{f(P)-f\left(F_{2}\right)}{\Delta t}, \quad\left(\frac{d f}{d t}\right)_{y_{3}}=\frac{f(P)-f\left(F_{3}\right)}{\Delta t},
\end{gathered}
$$

where $f$ is one of the functions $u, v, H$.

$E_{j}, F_{j}(j=1,2,3)$ are the points at the time step $t_{k}$

$P$ - the point at the time step $t_{k+1}$.

Substituting (3.1) into (2.4) we obtain:

$$
u(P)=\phi_{1} \Delta t+u\left(F_{1}\right)-u(M)+\frac{u\left(E_{2}\right)}{2}+\frac{C}{2 H} H\left(E_{2}\right)+\frac{u\left(E_{3}\right)}{2}-\frac{c}{2 H} H\left(E_{3}\right),
$$




$$
\begin{aligned}
v(P)= & \phi_{2} \Delta t+v\left(E_{1}\right)-v(M)+\frac{v\left(F_{2}\right)}{2}+\frac{c}{2 H} H\left(F_{2}\right)+\frac{v\left(F_{3}\right)}{2}-\frac{c}{2 H} H\left(F_{3}\right), \\
H(p)= & -H(M)+\frac{H}{2 c} u\left(E_{2}\right)+\frac{H\left(E_{2}\right)}{2}-\frac{H}{2 c} u\left(E_{3}\right)+\frac{H\left(E_{3}\right)}{2} \\
& +\frac{H}{2 c} v\left(F_{2}\right)+\frac{H\left(F_{2}\right)}{2}-\frac{H}{2 c} v\left(F_{3}\right)+\frac{H\left(F_{3}\right)}{2} .
\end{aligned}
$$

Assume that functions $u\left(x_{n}, y_{m}, t_{k}\right), v\left(x_{n}, y_{m}, t_{k}\right), H\left(x_{n}, y_{m}, t_{k}\right)$ at the time step $t_{k}$ are known. For determining these functions at the point $P$ of the time step $t_{k+1}$, we shall use the iterative method.

\section{1) For internal points}

i - Determine the coordinates of the points $E_{j}$ and $F_{j}(j=1,2,3)$ at the hyperplane $t_{k}$ (see Fig. 3)

$$
\begin{aligned}
& x_{E_{j}}=x_{M}+\frac{1}{2}\left[\left(\sigma_{j}\right)_{P}^{(i-1)}+\left(\sigma_{j}\right)_{E_{j}^{(i-1)}}\right] \Delta t, \\
& y_{F_{j}}=y_{M}+\frac{1}{2}\left[\left(\mu_{j}\right)_{P}^{(i-1)}+\left(\mu_{j}\right)_{F_{j}^{(i-1)}}\right] \Delta t,
\end{aligned}
$$

where $\left(\sigma_{j}\right)_{P}^{(i-1)}$ is the value $\sigma_{j}$ at the point $P$ at the iterative step $(i-1)$.

$\left(\sigma_{j}\right)_{E_{j}^{(i-1)}}$ - the value $\sigma_{j}$ at the point $E_{j}^{(i-1)}$ at the iterative step $(i-1)^{t h}$.

ii - Calculate the values of the functions $u, v, H$ at the points $E_{j}^{(i-1)}$ and $F_{j}^{(i-1)}$ by the linear interpolation method.

iii - Determine the values of functions $u, v, H$ at the point $P$ by formula (3.2).

The iterative process will be stoped, if the differences between values of these functions at the $i^{\text {th }}$ and $(i-1)^{\text {th }}$ iterative steps are small enough.

\section{2) For boundary points}

i - Linearising the boundary conditions and approximating the suitable supplementary equations from (2.5)-(2.10) by the difference expression (3.1), we can obtain a linear algebraic equation system, called $(3.2)_{B}$.

ii - Determine the points of intersection of the directions $x_{j}, y_{j}$ lying in the supplementary equations and the hyperplane $t=t_{k}$.

iii - Calculate the values $u, v, H$ at these points of intersection by the linear interpolation method.

iv - Substituting these values $u, v, H$ into $(3.2)_{B}$, we will obtain the equations at the boundary point for the time step $t=t_{k+1}$.

\section{Remark}

1) For the case, when the flow at the boundary is very slow $\left(w_{n} \ll 1\right)$ and it is not clear if there is inflow or outflow, we shall use the modifying Saint-Venant 
equation system (1.6). In this case, the supplementary equations will be two suitable equations describing in $(2.11) \div(2.16)$ and the subsequent computational process is analogous to the previous one.

2) In order to have difference scheme of high order of the accuracy, all coefficients $\left(\frac{H}{2 c}\right)$ and $\left(\frac{c}{2 H}\right)$ of the components $\varphi\left(Q_{j}\right)$ and $\phi$ in the equation system (3.2) must be of the following forms:

$$
\begin{aligned}
\psi\left(Q_{j}^{(i)}\right) & =\frac{1}{2}\left[\psi\left(P^{(i-1)}\right)+\psi\left(Q_{j}^{(i-1)}\right)\right], \\
\phi\left(P^{(i)}\right) & =\frac{1}{2}\left[\phi\left(P^{(i-1)}\right)+\phi(M)\right],
\end{aligned}
$$

where $\varphi$ is one of the functions $u, v, H$

$\psi$ - one of coefficients $\left(\frac{H}{2 c}\right),\left(\frac{c}{2 H}\right)$,

$Q_{j}^{(i)}$ - point $E_{j}^{(i)}$ or $F_{j}^{(i)}$ at the $(i)^{\text {th }}$ iterative step.

3) For the general equation system containing turbulent diffusion components

$$
\begin{aligned}
& \frac{\partial u}{\partial t}+u \frac{\partial u}{\partial x}+v \frac{\partial u}{\partial y}+g \frac{\partial H}{\partial x}=\phi_{1}+\nu \Delta u \\
& \frac{\partial v}{\partial t}+u \frac{\partial v}{\partial x}+v \frac{\partial v}{\partial y}+g \frac{\partial H}{\partial y}=\phi_{2}+\nu \Delta v \\
& \frac{\partial H}{\partial t}+\frac{\partial H u}{\partial x}+\frac{\partial H v}{\partial y}=0
\end{aligned}
$$

where:

$$
\Delta f=\frac{\partial^{2} f}{\partial x^{2}}+\frac{\partial^{2} f}{\partial y^{2}}
$$

$\nu$ is viscous coefficient;

Process of the computation will contain two steps:

a) Resolve the equation system (1.1) without turbulent diffusion components by the above method.

b) Determine the diffusion process by explicit scheme:

$$
\begin{aligned}
& \frac{\partial u}{\partial t}=\nu\left(\frac{\partial^{2} u}{\partial x^{2}}+\frac{\partial^{2} u}{\partial y^{2}}\right), \\
& \frac{\partial v}{\partial t}=\nu\left(\frac{\partial^{2} v}{\partial x^{2}}+\frac{\partial^{2} v}{\partial y^{2}}\right) .
\end{aligned}
$$

\section{Numerical experiments}

The algorithm is applied for solving following problems with $\nu=\frac{\alpha(\Delta x)^{2}}{2 \Delta t}$. 


\section{1) Test problem (see [7])}

A canal of $30 \mathrm{~m}$ long and $4 \mathrm{~m}$ wide connects with a basin of the area $15 \mathrm{~m} \times 14 \mathrm{~m}$ (see Fig. 4)

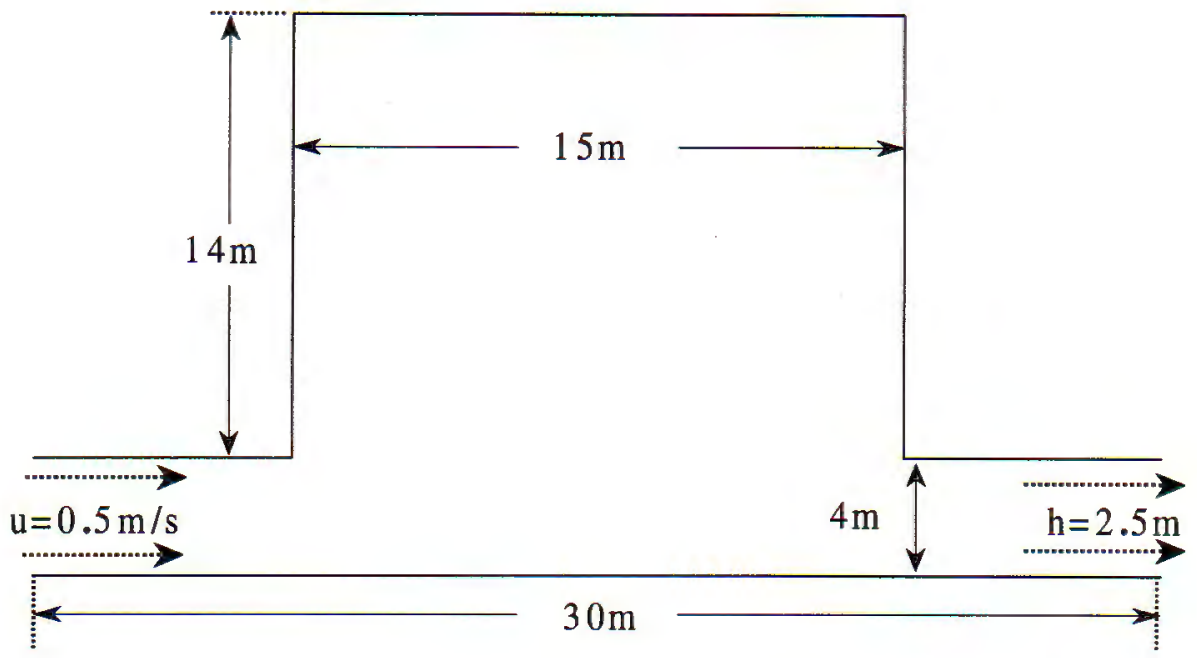

Fig. 4

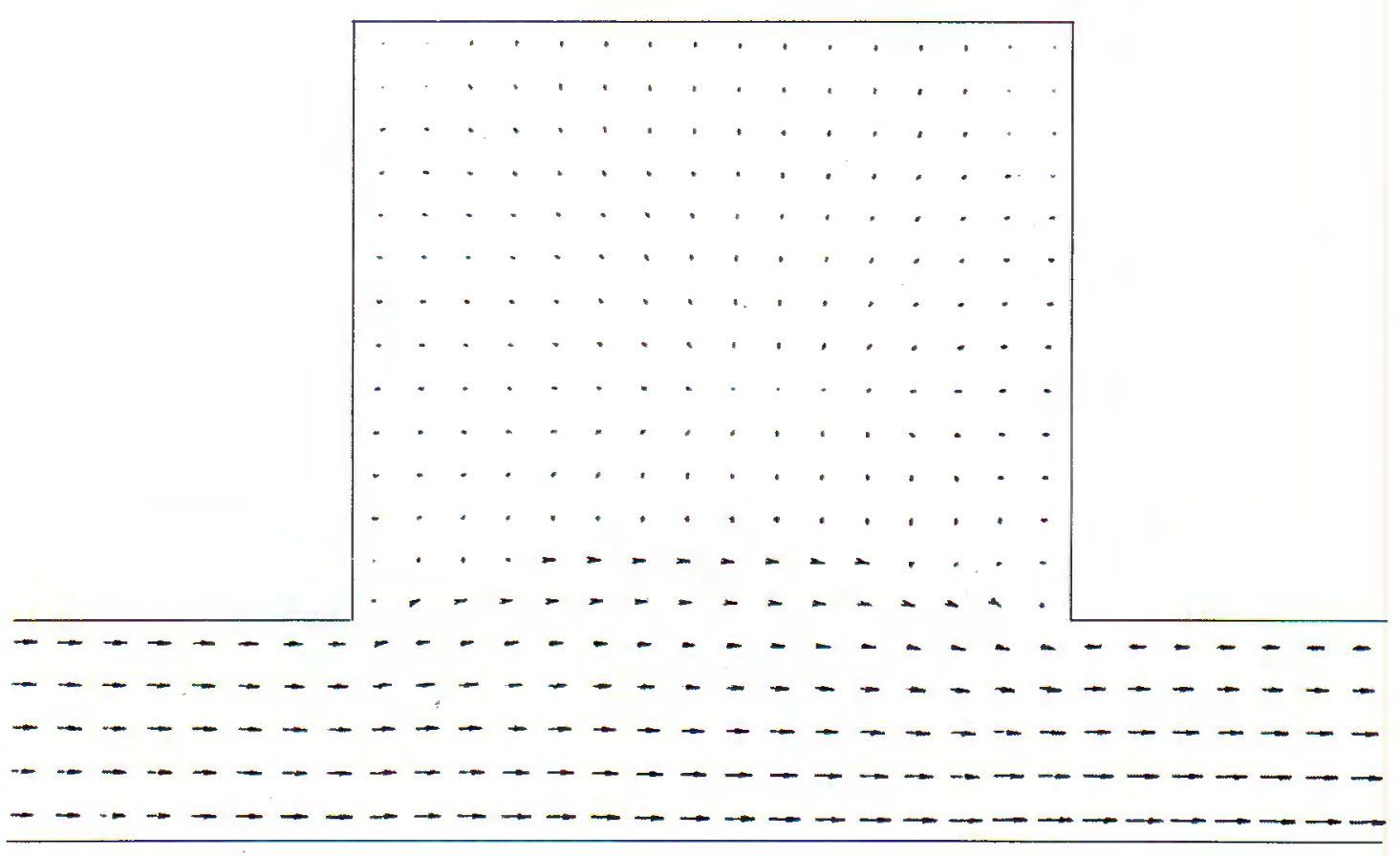

Fig. 5. Velocity field in the case $\alpha=0.0$

The bottom of the canal and the basin is horizontal and initial water depth is $2.5 \mathrm{~m}$. 
Left boundary condition; $u=0.5 \mathrm{~m} / \mathrm{s}$

Right boundary condition: $H=2.5 \mathrm{~m}$.

Computational results are shown on the Fig. 5 for $\alpha=0$ and Fig. 6 for $\alpha=0.1$.

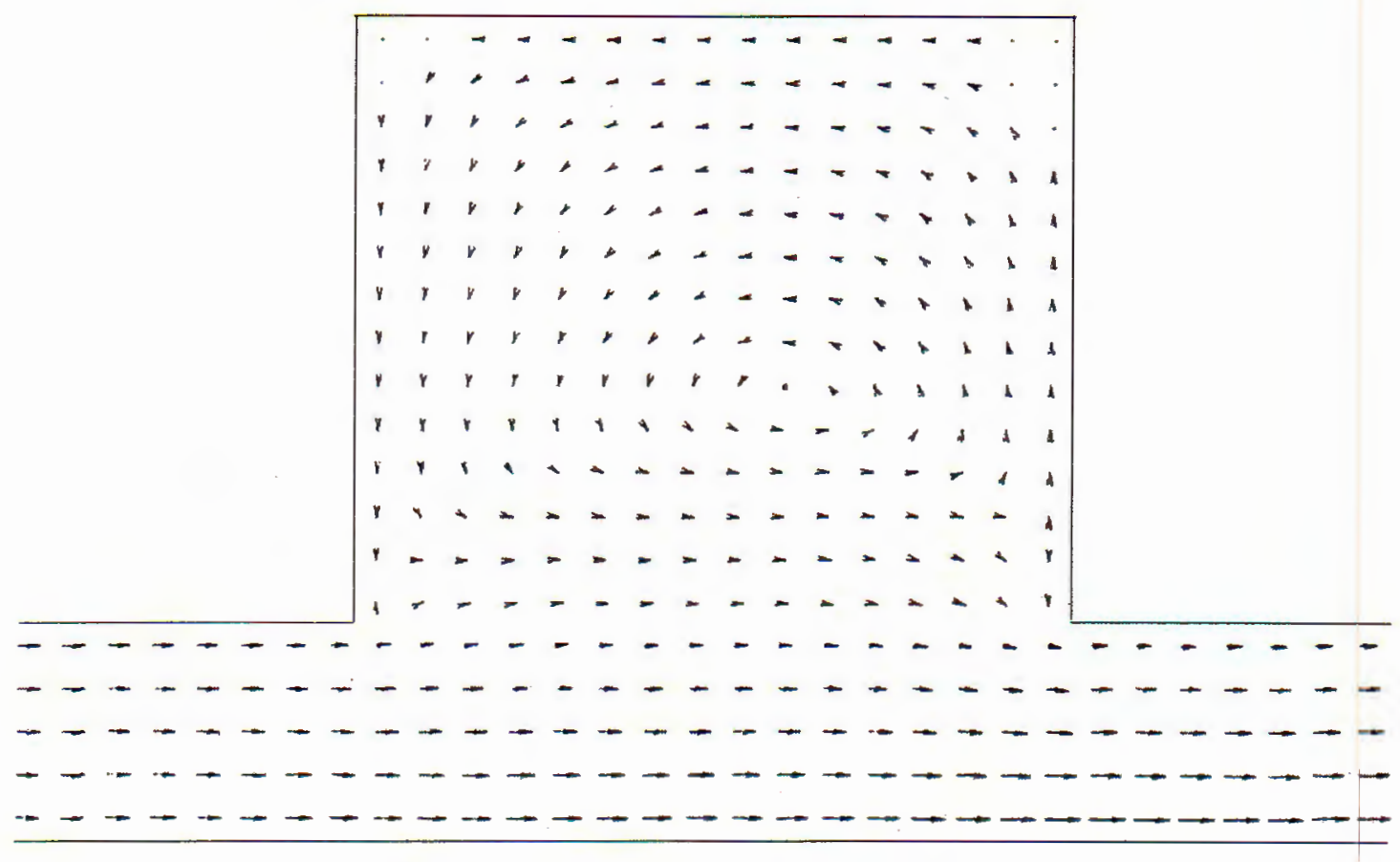

Fig. 6. Velocity field in the cases $\alpha=0.1$

They are coincided with the results obtained in [7].

\section{2) Second problem}

A canal of $10 \mathrm{~m}$ long and $2 \mathrm{~m}$ wide is given, the bottom is horizontal (see Fig. 7)

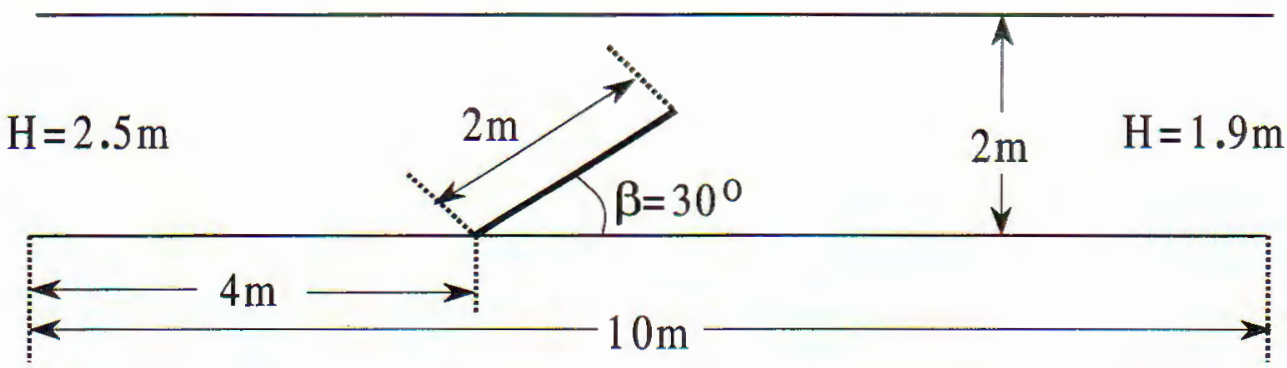

Fig. 7

Left boundary condition: $H=2.5 \mathrm{~m}$

Right boundary condition: $H=1.9 \mathrm{~m}$

There is a sluice $2 \mathrm{~m}$ long at the distance $4 \mathrm{~m}$ from left boundary making with the canal bank the angles $\beta=30^{\circ}$ or $\beta=45^{\circ}$. 


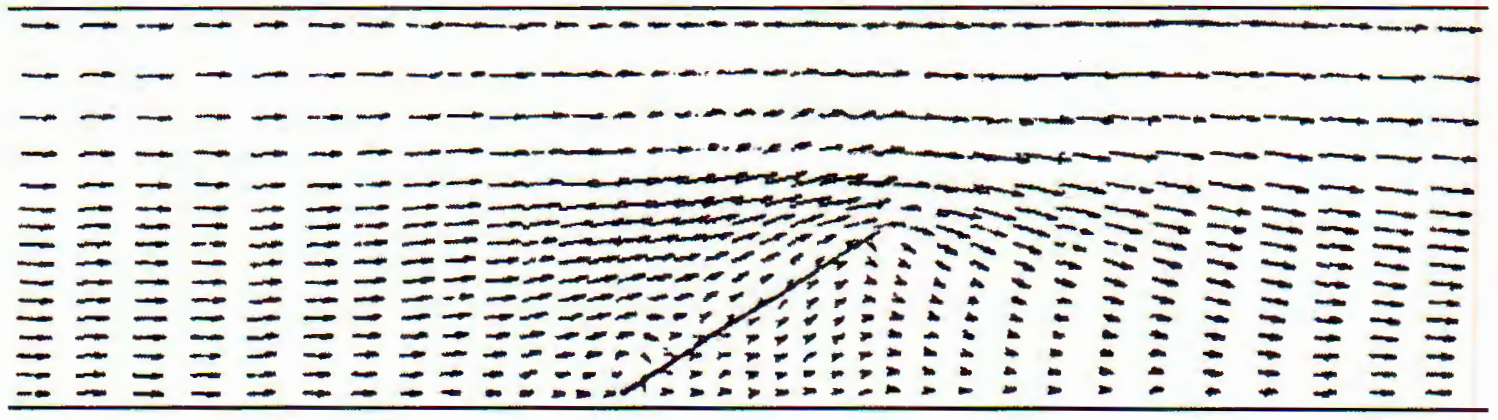

Fig. 8. Velocity field in the case $\alpha=0.0$ and $\beta=30^{\circ}$

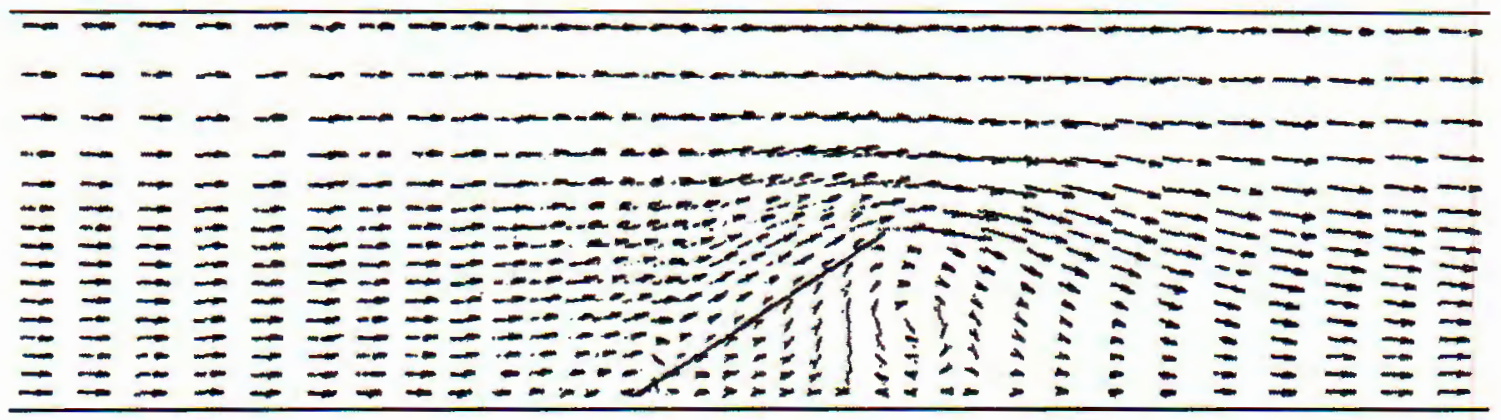

Fig. 9. Velocity field in the case $\alpha=0.1$ and $\beta=30^{\circ}$

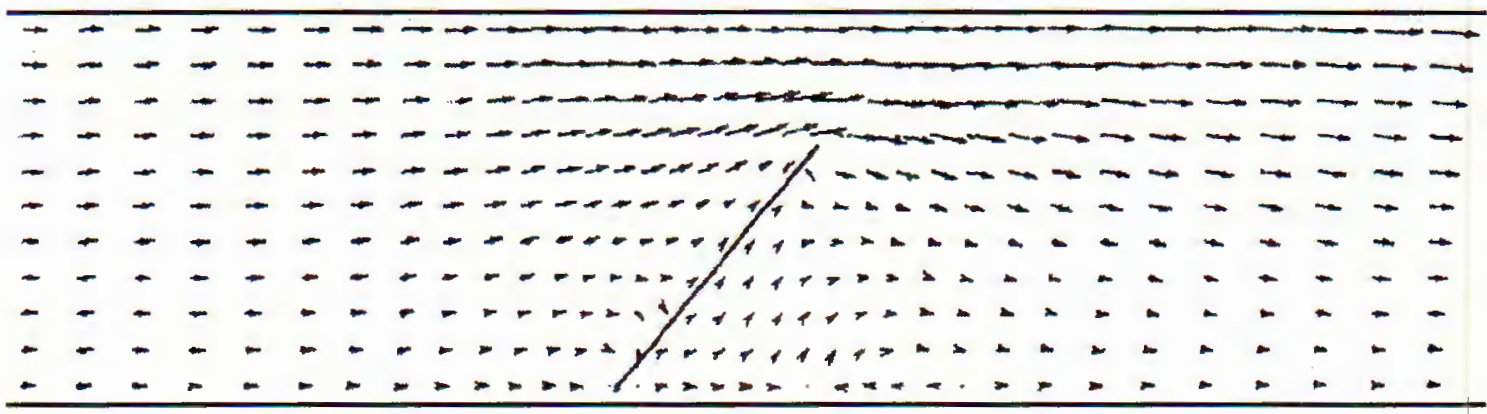

Fig. 10. Velocity field in the case $\alpha=0.0$ and $\beta=45^{\circ}$

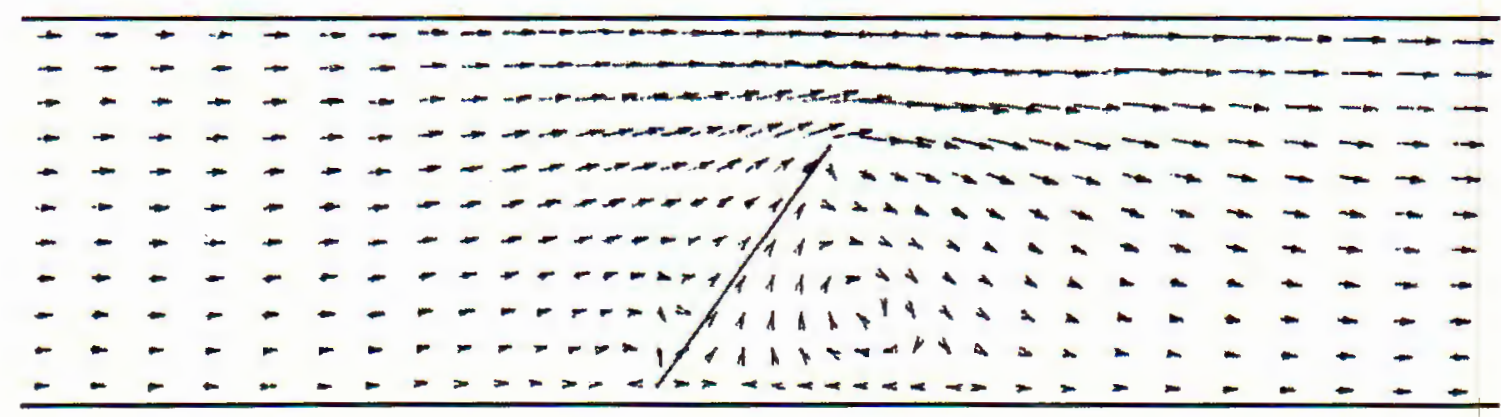

Fig. 11. Velocity field in the case $\alpha=0.1$ and $\beta=45^{\circ}$ 
Computational results are shown on Fig. 8 with coefficient $\alpha=0.0, \beta=30^{\circ}$ and Fig. 9 with $\alpha=0.1, \beta=30^{\circ}$; Fig. 10 with $\alpha=0.0, \beta=45^{\circ}$ and Fig. 11 with $\alpha=0.1$, $\beta=45^{\circ}$.

This work is supported by the Program of Basic Research in Natural Science.

\section{REFERENCES}

1. Voltsinger N. E., Pjaskovski R. V. Theory of the shallow water, Hydrometeopublishers, Leningrad, 1997.

2. Baklanovaskaja V. F. et all. A boundary problem for the Saint-Venant equation system on the plane, ZVM and MF, 19, No 3, 1978, p.708-724.

3. Nekrasov V. V. Standard procedure for integrating quasilinear partial differential equation system of hyperbolic type, ZVM and MF 20, No 2, 1980, p. 359-370.

4. Zauer R. Unsteady problems of gas dynamics, Publishers Mir, Moscow, 1967.

5. Courant R. Partial differential Equation, Publisheres Mir, Moscow 1964.

6. Godunov S. K. Equation of Mathematical-Physics, Publishers Nauka, Moscow, 1979.

7. Victor M. Ponce M. Asce and Steven B. Yabusaki. Modeling circulation in DepthAveraged flow. ASCE 107, No HY 11, November 1981, 1501-1516.

8. Tran Gia Lich - Le Kim Luat. Boundary conditions for the two-dimensional Saint-Venant equation system. Appl. Math. Modelling, 16, September 1992, pp. 498-502.

Received August 20, 2002

\section{TÍNH DÒNG CHẢY KHÔNG DƯNG HAI CHIÊUU NGANG BẰNG PHUƠNG PHÁP ĐẶC TRUNG}

Bài báo trình bày các vấn đề sau

* Hệ phương trình Saint-Venant hai chiều ngang dưới dạng dặc trưng

* Các phương trình bổ xung trên biên để kết hợp với các điều kiện biên tạo thành hệ phương trình khép kín xác định các giá trị nghiệm trên biên

* Phương pháp đặc trưng tính dòng cháy không dừng hai chiều ngang

* Tính toán thử nghiệm với hai bài toán cụ thể. 